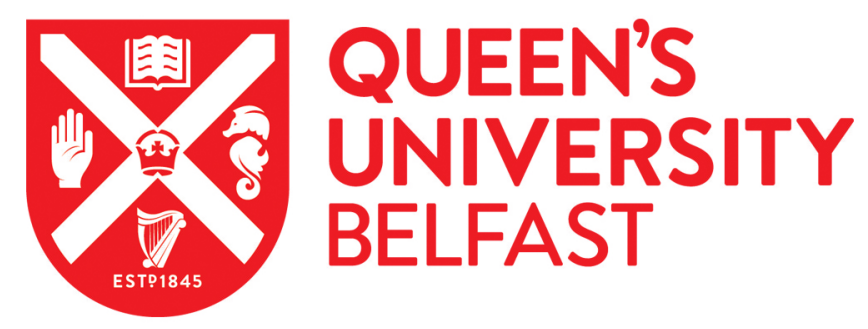

\title{
Fast and Energy-Efficient OLAP Data Management on Hybrid Main Memory Systems
}

Hassan, A., Nikolopoulos, D., \& Vandierendonck, H. (2019). Fast and Energy-Efficient OLAP Data Management on Hybrid Main Memory Systems. IEEE Transactions on Computers. https://doi.org/10.1109/TC.2019.2919287

\author{
Published in: \\ IEEE Transactions on Computers
}

Document Version:

Peer reviewed version

Queen's University Belfast - Research Portal:

Link to publication record in Queen's University Belfast Research Portal

Publisher rights

(C) 2019 IEEE. This work is made available online in accordance with the publisher's policies. Please refer to any applicable terms of use of the publisher.

\section{General rights}

Copyright for the publications made accessible via the Queen's University Belfast Research Portal is retained by the author(s) and / or other copyright owners and it is a condition of accessing these publications that users recognise and abide by the legal requirements associated with these rights.

Take down policy

The Research Portal is Queen's institutional repository that provides access to Queen's research output. Every effort has been made to ensure that content in the Research Portal does not infringe any person's rights, or applicable UK laws. If you discover content in the Research Portal that you believe breaches copyright or violates any law, please contact openaccess@qub.ac.uk. 


\title{
Fast and Energy-Efficient OLAP Data Management on Hybrid Main Memory Systems
}

\author{
Ahmad Hassan, Dimitrios S. Nikolopoulos, Senior Member, IEEE, \\ and Hans Vandierendonck, Senior Member, IEEE,
}

\begin{abstract}
This paper studies the problem of efficiently utilizing hybrid memory systems, consisting of both Dynamic Random Access Memory (DRAM) and novel Non-Volatile Memory (NVM) in database management systems (DBMS) for online analytical processing (OLAP) workloads. We present a methodology to determine the database operators that are responsible for most main memory accesses. Our analysis uses both cost models and empirical measurements. We develop heuristic decision procedures to allocate data in hybrid memory at the time that the data buffers are allocated, depending on the expected memory access frequency. We implement these heuristics in the MonetDB column-oriented database and demonstrate performance improvement and energy-efficiency as compared to state-of-the-art application-agnostic hybrid memory management techniques.
\end{abstract}

Index Terms-Non-volatile memory, hybrid main memory, database management system

\section{INTRODUCTION}

Online analytical processing (OLAP) is a key component of modern data processing pipelines and aims at analyzing large data sets to inform business decisions. OLAP consists of long-running, complex transactions that are readintensive. To maintain high processing throughput, data sets are kept in main memory during OLAP processing to avoid I/O bottlenecks. This approach, known as in-memory processing, puts severe pressure on the required main memory capacity as data sets are continuously growing. It is estimated that data sets world-wide double in size every year [20]. As such, multi-terabyte main memory setups are desired [48].

DRAM, the dominant technology for main memory chips, has hit power and scaling limitations [22], [64]. DRAM-based main memory consumes $30-40 \%$ of the total server power due to leakage and refresh power consumption [3], [30], [34]. The background power consumption of DRAM moreover scales proportionally with the DRAM size, adding to the total cost of ownership (TCO). Moreover, it is uncertain whether DRAM technology can be scaled below $40 \mathrm{~nm}$ feature sizes [22], [64]. It is thus unlikely that DRAM will continue to be the dominant memory technology. The techniques like High-Bandwidth Memory (HBM) and Intel's Knight's Landing processor provide ways of mitigating DRAM limitations [25] [53].

An alternative setup uses a combination of conventional DRAM and novel byte-addressable non-volatile memory (NVM) chips that are interface-compatible with DRAM [27], [28], [46], [47]. Emerging NVM technologies such as PhaseChange Memory (PCM), Spin Transfer Torque RAM (STT-

- Ahmad Hassan is with SAP. He was with Queen's University Belfast, United Kingdom at the time this research was conducted.

E-mail:ahmad.hassan@sap.com

- Dimitrios S. Nikolopoulos and Hans Vandierendonck are with Queen's University Belfast.

Manuscript received June 6, 2018; revised December 2, 2018.
RAM) and Resistive RAM (RRAM) [19], are compelling alternatives due to their high density and near-zero leakage power [8], [13], [37]. NVM technologies however have several drawbacks, broadly involving increased latency and increased dynamic energy for NVM accesses, reduced memory bandwidth and a fast wear-out of memory cells [8], [28], [46], [65]. On the plus side, NVM is predicted to scale to smaller feature sizes [8] and has around $100 \times$ lower static energy due to the absence of refresh operations [13]. Moreover, NVM is persistent, which is important to the design of DBMSs. This work, however, focuses on the orthogonal aspects of energy-efficiency and performance.

Hybrid memory systems typically have a fast and small component and a large and slow component, each with distinct energy consumption. In the case of a DRAM+NVM hybrid memory, DRAM provides high performance but it is not feasible to have large amounts of it. On the other hand, NVM can be scaled up to provide large amounts of memory, but it cannot be accessed as efficiently as DRAM. Hybrid memory systems raise the key question of how to distribute the data over the memory types?

State-of-the-art techniques propose data migration policies for hybrid memory where data migration is decided on by the hardware [28], [46], [53], [62], the operating system [12], [50] or a mixture of both [47]. These techniques try to second-guess the properties of the workload and migrate large chunks of data, typically at the page granularity of the virtual memory system. This reactive approach introduces runtime overhead and energy consumption due to monitoring and migration.

In this paper, we propose a radically different approach: Building on intricate knowledge of the structure and memory access patterns of column-oriented database, we propose that the DBMS manages data placement in the hybrid main memory. We present a methodology to analyze a DBMS in order to identify a good strategy for data placement. Our analysis uses a tool [18] that instruments all program variables and memory allocations and collects statistics on 
their size, their lifetime and on the number of memory accesses made for each. Using these statistics, we formulate a strategy for data placement in a hybrid main memory system. In our solution the DBMS analyzes the query plan to predict which data sets will incur frequent memory accesses and should thus be placed on DRAM. It then allocates these data sets on DRAM as they are generated. By predicting the best placement with high accuracy, our solution does not require migration of data between DRAM and NVM to fix wrong data placements. This contributes to its performance and energy-efficiency.

The key contributions of this paper are:

- Derivation of runtime data placement heuristics for inputs and intermediate allocations of hash join using the access patterns of binary algebraic operators. The hash join is the single most important operation in our OLAP workloads. Our solution, optimized for OLAP workloads, requires only two heuristics for MonetDB [6], a state-of-the-art column-oriented database. Our approach is, however, not limited to MonetDB.

- Demonstrating the performance and energyefficiency of our data placement heuristics for hybrid memory in MonetDB. We furthermore compare our heuristics to state-of-the-art application-agnostic page placement and migration techniques.

- Demonstrating that query plan partitioning yields intermediate data sets that are small enough to fit in DRAM without incurring significant overhead.

The remainder of this paper is organized as follows. Section 2 reviews the organization of memory systems and their key performance and energy properties. Section 3 describes the system organization assumed in this work and the programming interface. Section 4 analyzes what algebraic operators cause frequent memory accesses. Section 5 develops data placement heuristics for the most memoryintensive algebraic operators. Section 6 experimentally evaluates our solution.

\section{Background and Related Work}

\subsection{Column-Oriented Databases}

A column-oriented database stores relational tables as a collection of individual columns rather than as a collection of rows [7], [55]. The columnar layout has proven specifically efficient on large, read-mostly data sets and on analytical workloads such as online analytical processing (OLAP) [7], [21]. The benefits of columnar databases arise from loading only relevant fields of tables into CPU caches. Fields that are not required for a query are stored on separate columns and are not accessed during evaluation of the query.

Similar to record-oriented databases, columnar databases break down SQL queries in a query execution plan that explains how to evaluate the query using elementary operations (the algebra) as a function of the database state. A binary algebra [6] applies algebraic operations such as select (filter), join, union, etc, to two columnar data sets. The query optimizer transforms the initial query plan into an equivalent plan that is expected to execute faster. The query optimizer relies on heuristics, e.g., to apply filter operations as early in the query plan as possible in order to reduce data volumes quickly.

Columnar databases require materialization, which is the act of "gluing" together the columns that make up a relation [1]. Early materialization glues together all columns that are needed in the evaluation of the query. This then is similar to executing the query in the same style as a recordoriented database where records are initially shortened to contain only the columns relevant to the query. Late materialization postpones glueing together the columns as long as possible. Algebraic operators are not applied to the data columns themselves. Instead they operate on columns that hold the indices of the relevant records.

We focus our work on MonetDB [6], a highly efficient columnar database. MonetDB uses late materialization, which results in high memory locality [6]. Moreover, MonetDB evaluates each operator at a time, i.e., it processes the full input columns to produce the output(s) before proceeding to evaluate the next operator.

\subsection{The Memory Hierarchy}

The goal of cache memories (or caches for short) is to create the impression of a large and fast memory, where in reality large memories are slow and fast memories must be small. Cache memories create this impression using the principle of reference locality, an empirical observation that programs tend to repeatedly access only a limited amount of data during any period of time. This limited amount of data is called the working set of the program. Only the first access to the working set is slow. Subsequent accesses are fast as the working set is then available in the cache. We call a cache access that finds the data in the cache a cache hit. A cache access failing to find the data is a cache miss.

Caches are typically organized in multiple levels to form a cache memory hierarchy. These caches are searched one after the other until the data is found. The search starts with the cache closest to the CPU, which we also call the level 1 cache. This cache is the smallest and fastest. Subsequent levels are numbered accordingly as level 2 and level 3 cache and are increasingly larger and slower. The last level of cache is conveniently referred to as the last level cache or LLC.

When a cache miss occurs in the LLC, the missing cache block is fetched from main memory. Main memory accesses are off-chip, implying a high performance penalty in comparison to an on-chip cache hit. In the remainder of this work, we will focus on LLC cache misses, i.e., those load/store instructions that result in actual memory accesses.

When data is retained in on-chip caches, load/store instructions are served from the cached data and do not require main memory accesses. Consequently, small objects, which tend to be maintained in on-chip caches, may be allocated on NVM. This is true even if they are frequently accessed.

The replacement policy determines which data is maintained in the last-level cache, and which is evicted [52]. The least recently used (LRU) replacement is a common policy that replaces the least recently used cache block [52] Instead of moving a block to the most recently used position 
TABLE 1: Latency (ns) and dynamic energy (nJ) for a 64-byte access and leakage power (mW/GB) for NVM technologies.

\begin{tabular}{|c|c|c|c|}
\hline & Latency & Dyn. Energy & Leakage \\
\hline & R/W, [ns] & R/W, [nJ] & [mW/GB] \\
\hline DRAM & $60 / 60[35]$ & $11.8 / 25.4[35]$ & $451[35]$ \\
\hline PCM & $302.8 / 791.8[14]$ & $160.82 / 837.2[14]$ & $4.23[13]$ \\
\hline STT-RAM & $210 / 154.42[14]$ & $43.4 / 53.21[14]$ & $4.23[13]$ \\
\hline RRAM & $124 / 151[14]$ & $15.88 / 30.24[14]$ & $4.23[13]$ \\
\hline
\end{tabular}

upon access, it is proposed to insert it in the LRU position, or in a median position [24], [45]. Adaptive policies select different insertion positions for workloads with mainly capacity misses compared to those with mainly conflict misses [24], [45]. While these policies aim to minimize cache miss rates, replacement policies have been proposed that minimize write-back traffic [15], [60]. These techniques are orthogonal to the present work as we aim to allocate data on appropriate memory types based on estimated capacity misses.

\subsection{Memory Access Costs}

Memory accesses incur costs in performance as well as in energy. These properties are relevant to all caches and also for main memory.

Latency is the time that elapses between initiating a data access and the result returning in the CPU. Latency increases as data is found in cache levels further away from the CPU and increases sharply when main memory is accessed.

Bandwidth is the volume of data that can be transferred per unit of time. Bandwidth decreases as one moves away further from the CPU. Moreover, DRAM has higher bandwidth than NVM.

Static energy, also called background energy, is consumed continuously regardless of whether the memory is accessed by software. Static energy consists of leakage energy in the memory cells and in peripheral logic. In DRAM, the memory cells must be refreshed frequently due to leakage. This contributes also to the static energy.

Dynamic energy is incurred on a memory access. Dynamic energy consists of multiple components, depending on the state of the memory. It includes transfer of a command over the bus, opening a page, i.e., reading the contents of a row of memory cells into the row buffer, and selecting and sending data from the row buffer over the bus. After some time has elapsed, pages are closed which implies that the row buffer contents are written back to the actual memory cells, if modified.

It is reasonably accurate to reason about static and dynamic energy consumption of main memory using the capacity of the memory and the volume of data transferred [54].

\subsection{Non-Volatile Main Memory Technologies}

Table 1 shows key performance and energy consumption characteristics of some NVM technologies that are relatively close to market, i.e., they are in a good state of maturity and expected to ship in volume in the next few years. As such, precise characteristics of the memory chips are still unknown. However, regardless of the technology, it may be expected that reading and writing will take longer for NVM than for DRAM and will consume more energy, with writing suffering more than reading [28], [64]. Consequently, NVM is not a drop-in replacement for DRAM.

This paper focuses on the difference in memory access latency and bandwidth, as well as the trade-off between static and dynamic energy. These properties dictate that NVM can be used to build a large memory (due to the low static energy) but accessing the data stored in NVM is expensive both in time and energy. In contrast, DRAM can be accessed at a higher rate with less energy. However, building a large DRAM memory consumes a prohibitive amount of energy due to leakage and refresh operations.

These differences motivate the design of a hybrid main memory, featuring a relatively small DRAM component where the majority of read and write activity should occur, and a large NVM component where reads and writes are less frequent. DRAM thus gravitates towards the role of a cache, which is designed to hold the working set of an application.

\subsection{Hybrid Memory Organization Options}

CPU caches are typically physically organized as a hierarchy of caches where the highest-level cache is connected directly to the CPU data path using a wide and fast bus. The secondlevel cache is connected to the controller of the highestlevel cache and is accessible only indirectly in the event of a cache miss in the highest-level cache. In contrast, in a hybrid memory system, DRAM and NVM are physically organized side-by-side and are connected to the CPU using a bus in a physically symmetric way. Nonetheless, DRAM can functionally be organized as a cache, where cache placement and replacement policies are in effect to control data allocation in DRAM (placement) and to evict data from DRAM (replacement).

A DRAM cache is governed by the same parameters and properties as CPU caches. However, data is moved in or out at a larger granularity. Typically the block size of a DRAM cache is comparable to the virtual memory page size. The DRAM cache can be managed either using hardware implementation of the placement and replacement policies, implementation of those policies in the operating system or in application software.

Hardware cache management implies that a hardware controller decides when to migrate pages between DRAM and NVM. Page migrations are expensive in both time and energy, which implies they must be performed with care. For instance, Lee et al [28] buffer a sequence of CPU writes to the same memory locations in DRAM before updating the main copy in NVM. This mitigates the high cost of NVM writes. Yoon et al [62] store those rows of data in DRAM that incur frequent misses in PCM row-buffers. They predict hot PCM rows that cause frequent row-buffer misses and move these rows to DRAM cache. Such caching policy shows $14 \%$ performance improvement and energy savings up to $10 \%$. Their approach requires learning to predict hot data. These techniques require a directory to determine where data blocks are stored, which adds complexity.

Operating systems can implement page migration algorithms to decide which pages should be migrated to/from DRAM and NVM. These techniques require that the hardware monitors the frequency of memory accesses for each 
page. Dhiman et al [12] show energy savings up to $30 \%$ and performance degradation up to $6 \%$ using these ideas. Shin et al [50] adaptively group pages with similar access characteristics. They migrate similar pages in group to reduce overheads and demonstrated average energy savings up to $36 \%$ and less than $8 \%$ performance performance overhead. Li et al [31] propose an OS and compiler-assisted approach to identify write intensive parts of the application. In their approach the compiler adds new instructions to the application to guide hardware data distribution in hybrid memories. Ramos et al [47] use multi-level queues to rank pages according to the benefit of storing them in DRAM. Their Rank-based Page Placement (RaPP) policy improves performance and reduces wear-out of NVM cells by minimizing the writes to NVM.

Application software can contribute to the placement and migration of data in hybrid memories. Effectively, the placement and replacement policies are now implemented in software in an application-specific way. From a hardware perspective, the DRAM and NVM components are now independently accessible. This moreover has the benefit that these policies can leverage insight in the future activity of the application provided that the application is well understood.

Wang et al [59] optimize data placement using integer linear programming in the context of a real-time multitasking system. While theoretically tractable, they assume that each task accesses only one type of memory. This is unnecessarily restrictive. In [17], the memcached key-value caching program is modified to decide data migrations. The authors extended memcached's replacement algorithm such that hot key-value pairs are retained in DRAM.

Hassan et al [18] use off-line profiling information for each variable or memory allocation, called objects, in the application to decide a static placement. They do not perform migration during execution as they observed that objects in their applications are typically too short-lived to warrant migration. Moreover, they argue that placing objects in hybrid memories is more effective than placing virtual memory pages as the latter often contain several objects with distinct memory access patterns. Wei et al [61] assume a similar setup but extend it with migration. They first place objects in the hybrid memory using the off-line profiled ratio of reads over writes for each object as a guideline. During execution, they again measure the read/write ratio and migrate pages as appropriate. The read/write ratio is however an inaccurate predictor for energy consumption as energy consumption relates linearly to the number of reads and writes [58]. It does not depend solely on the ratio of reads to writes.

This paper manages the DRAM cache from within the application, in our case the DBMS. When applicable, this approach has the highest potential. Application-agnostic techniques incur overhead to learn memory access patterns and migrate data between DRAM and NVM to correct a bad allocation. In contrast, we will demonstrate that we can accurately allocate data in a column-oriented DBMS immediately in the right type of memory by analyzing the query execution plan and estimating what operators will incur frequent memory accesses.

\subsection{Persistence Aspects of NVM}

The persistent nature of NVM is attractive to optimize transaction processing workloads. Coburn et al [11] and Guerra et al [16] provide programming API's with ACID properties applicable to generic data structures such as trees and hash tables. Jorge et al showed a speed-up of $83 \%$ for the SQLite application. Arulaj et al [2] investigate how three different DBMS designs can exploit the persistence of NVM. Pelley et al [42] reduce the frequency of persistence barriers by exploiting the byte-addressability of main memory NVM. We do not utilize the persistence properties of NVM in this work. Oukid et al [40] proposed a database storage engine on top of DRAM and NVM to achieve data recovery through NVM persistence. Similarly, Oukid et al [41] used NVM persistence to eliminate database logging mechanism in contrast to traditional database systems running on top of DRAM-only system. As such, the ideas above can be integrated with our techniques.

\subsection{NVM Durability}

Due to the changes in physical structure when writing NVM memory cells, NVM is typically less durable, i.e., it has a shorter lifetime than DRAM. The durability of NVM can be improved in various ways, including changes to the internal organization of memory chips [28], [46], encoding energy-efficient encoding of bit patterns based on the energy difference between writing a 0 (RESET) and a 1 (SET) [36], or by avoiding redundant writes where the new value equals the overwritten one [64]. These techniques will make NVM more attractive and less expensive. They are otherwise orthogonal to this work.

\section{System Organization and Programming INTERFACE}

The programming interface exposes the hybrid nature of the main memory (Figure 1) to the programmer [18]. At the hardware level, the DRAM and NVM memory chips are assigned distinct physical address regions. This ties in with how the BIOS reports DIMMs and their physical address ranges to the OS. In this case, the BIOS additionally reports the technology used to implement the DIMMs. The OS thus knows which memory chips are DRAM and which are NVM and can map virtual memory pages accordingly.

The operating system allows the application programmer to decide if data should be allocated on NVM or on DRAM. The main mechanism to create new virtual memory pages is through the mmap system call. We add a new option to the flag argument of mmap to indicate whether the new pages should be mapped on DRAM or on NVM (default). The OS records the target memory request and allocates the physical page on DRAM if space is available. If space is not available, the physical page is allocated on NVM.

Applications often use finer-grain mechanisms for memory allocation such as the malloc family of functions. The malloc functions manage a set of virtual memory pages obtained through mmap and allocate small objects from these pages. In order to differentiate between DRAM and NVM allocations, we implement two distinct memory allocators in the application, one managing pages mapped to DRAM, 


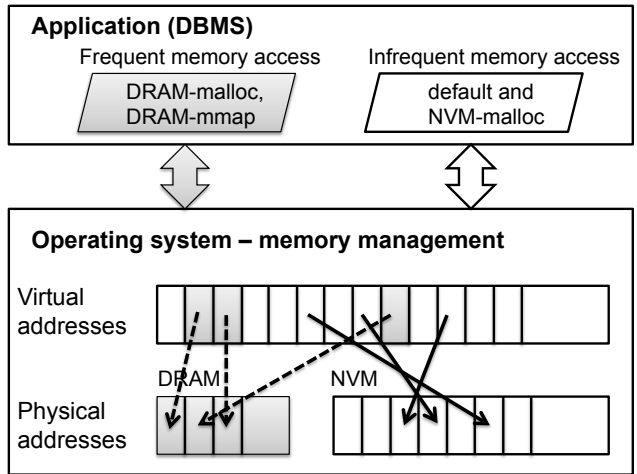

Fig. 1: Operating system interface for mapping data to hybrid memory.

the other pages mapped to NVM. Each memory allocator provides the standard memory allocation functions prefixed with either "dram_" (e.g., dram_malloc) or "nvm_". The standard function names are retained and are mapped to the NVM allocator.

The proposed solution is orthogonal to the Persistent Memory Programming (pmem.io), which exposes a set of user space libraries that provide APIs to allocate memory from within the applications while taking care of the persistence memory management [44]. Persistent memory builds on the Direct Access (DAX) feature of linux which enables to run load/store instructions on a memory mapped file. The solution proposed in this paper is an extension of pmem.io, where the application memory allocators are aware of the hybrid memory and pmem.io is used to support load/store instruction access to NVM.

For the purposes of this work, it suffices to consider only dynamically allocated memory through mmap or malloc. We assume that static (global) variables and stack-allocated variables are mapped to NVM. These variable are typically small and have good CPU cache locality [9], [29]. As such, few main memory accesses are directed to these variables and placing them in NVM is energy-efficient and does not incur important additional memory access latency.

If migration is necessary, the programmer can allocate a new copy of the object on the opposite memory type and copy the data. In this paper, we find that migrations always coincide with existing memory copying code. This happens as migrations are performed only when data set sizes grow to exceed the LLC capacity. As data set sizes are hard to predict in advance, data is stored in incrementally growing buffers. Growing these buffers involves allocating a new buffer and copying the data over. We leverage these existing copies to avoid additional migration cost.

\section{Analysis}

Our model for utilizing hybrid memory requires to identify specific memory allocations, through mmap or malloc, that should be placed on DRAM. These objects should have a high number of anticipated off-chip memory accesses. In order to determine these objects, we analyse what data and code in the DBMS is responsible for the majority of main memory accesses.
The analysis described in the current section is to a degree specific to the type of DBMS that we analyse. We analyse, specifically, MonetDB, a highly efficient columnoriented database [6], [38]. The technique of the analysis, however, applies without modification to other DMBS. Our analysis consists of the following steps, automated by a tool:

1) We perform dynamic analysis of the DBMS through instrumenting all memory access instructions in the executable. We execute the instrumented DBMS on real hardware and track all memory accesses, heap allocations and de-allocations and function calls and returns. This analysis identifies all memory objects in the application, which are program variables and (heap) memory allocations. The dynamic analysis is implemented through instrumentation of the DBMS with a custom LLVM pass [32].

2) We focus on the hot objects, those with the highest number of main memory accesses, as determined through CPU cache simulation during dynamic analysis. The cache simulator is a simple, singlelevel cache simulator that models a $20 \mathrm{MB}$ 4-way set-associate cache with 64-byte blocks and leastrecently used (LRU) replacement. The simulator makes the simplifying assumption that a hierarchy of inclusive caches may be approximated by a single cache level consisting of the LLC [52].

3) We analyse the code that is responsible for creating and accessing the hot objects, which is identified by the dynamic analysis tool.

\subsection{Column-Oriented Data Types}

We have applied our dynamic analysis tool to MonetDB and identified the following types of objects, all of which are dynamically allocated:

- Columns: These store the columns of the relational database as it is mapped on the physical storage. All data within these data structures are backed by files on the physical storage. There is relatively little reuse in columns as they are mostly touched once per query in analytical workloads.

- Intermediate data: These are intermediate and final results sets that are computed by operators in the query execution plan. Intermediate result sets require further processing by operators in the query execution plan. Many small intermediate data sets are generated during execution of a query.

- Schema Metadata: These are database columns holding meta-data on the schema stored in the database. As the metadata describes the structure of the database, its volume is typically small in relation to the contents of the database.

DBMSs perform numerous other memory allocations. Any memory allocation not listed above has marginal relevance to the volume of memory addressed and the total number of off-chip memory accesses made.

\subsection{Frequently Accessed Objects}

We analyzed all objects created by MonetDB while executing the TPC-H queries. Objects incurring frequent main memory accesses should be placed on DRAM, while objects that 


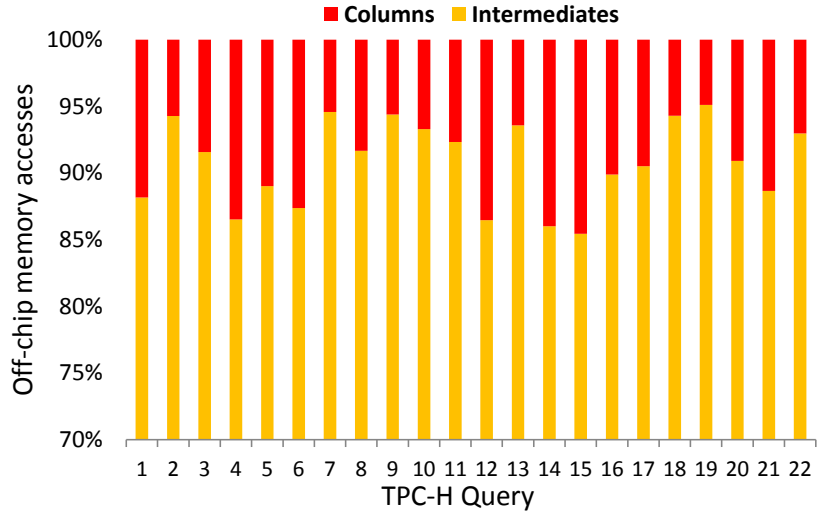

Fig. 2: Percentage of off-chip accesses to column and intermediate data sets for each of the 22 TPC-H queries. Note the scale starts at $70 \%$.

are infrequently read or written in main memory should be stored in NVM. Note that this property is not the same as frequency of load and store operations at the application level. The CPU caches filter out most of the load and store operations, such that only a small percentage actually reaches main memory. As such, our analysis depends on system properties. We assume that modeling only the lastlevel cache provides an accurate depiction of the cache hits and misses that would occur in a more detailed memory hierarchy with higher-level caches. This assumption results in sufficient accuracy for the purposes of our analysis. Moreover, it results in a significant speedup of profiling which allows us to profile the complete execution of the database. We assume a $20 \mathrm{MB}$ last-level cache. We analyse MonetDB version 11.15.7 executing the TPC-H workload at scale factor 5.0. We have analyzed also scale factors up to 100 and remark that the conclusions presented here are valid at least up to scale factor 100 .

Our measurements confirm that over $99 \%$ of the loads and stores are issued on the database objects listed in Section 4.1. These objects have, however, distinct characteristics. Some objects have very good cache locality while others have few overall accesses. We distinguish between two types of objects: objects storing the columns of the database and objects storing intermediate results. Figure 2 shows that the majority of off-chip accesses are incurred for intermediate results. The actual columns contribute on average to less than $10 \%$ of the main memory accesses. This is a logical consequence of query plan optimization where cache-efficient operators such as select are performed early on in order to reduce intermediate data set sizes. As such, columns tend to be accessed in a cache-efficient way.

Implications for placement: Most objects in MonetDB benefit from a default placement on NVM. E.g., objects storing schema metadata tend to be small and are accessed primarily during the construction of the query plan.

Our analysis shows that columns may be mapped to NVM with limited overhead. This is a helpful result because the columns hold the actual data in the database. As such, they may be very large compared to the available DRAM.

Objects holding intermediate data should be distributed between DRAM and NVM, as only a subset of these objects incur a high number of main memory accesses. Note that there is no guarantee that intermediate objects may actually fit within the amount of DRAM available. However, the sizes of intermediate data sets can be controlled by fragmenting the query execution plan [23]. We will demonstrate that this allows us to make excellent use of available DRAM.

\subsection{Operators Causing Main Memory Accesses}

Now we know what objects cause main memory accesses, we turn to the question what code is making those main memory accesses. We calculated a break-down of main memory accesses by type of operator executed. Because the results are highly skewed, we report results only on the join operators. Figure 3 shows what percentage of main memory accesses are incurred by join operations versus other database code. Over $90 \%$ of the main memory accesses are incurred by join operations. This result is in line with previous observations that join operations are the most critical and problematic database operations [7].

Figure 3 also shows the impact of query plan fragmentation. Query plan fragmentation is a technique to enlarge the degree of parallelism within the query plan [23]. It breaks down the columns in smaller fragments, resulting in more operations in the query plan that are independent of one another. In this work, we will use query plan fragmentation to constrain the size of intermediate result sets such that they fit in DRAM. Note that other databases may use other techniques to control parallelism and working set sizes. Our key interest is in limiting intermediate data set sizes such that they fit in DRAM. Figure 3 shows that breaking down columns in 50 fragments slightly reduces the percentage of main memory accesses concentrated in join operations. This effect is, however, negligible. Figure 4 shows that, for the 5 GB database, up to 150 fragments incur no noticeable performance overhead. The baseline is no fragmentation. At 200 fragments, the overhead increases sharply due to an increased number of operators in the query execution plan.

Practically, fragmentation is applied by identifying a limit to the size of partitions of columns. Fifty-way fragmentation results in fragments of columns of the TPC-H data set at scale factor 5 no larger than $80 \mathrm{MB}$. As such, 4 column fragment fit simultaneously in a 256 MB DRAM cache, which is the size assumed in our experimental evaluation. More importantly, intermediate data sets are typically much smaller, so many more can fit simultaneously in the DRAM cache.

Modern databases implement various join algorithms, each optimized to different boundary conditions [49]. The three most frequently occurring join operations in our analysis are: the fetch join (a selection-based join able to join tables with a single pass), the hash join (which summarizes the smaller relation and joins through lookup in the hash table) and merge join (which merges relations that are sorted on the join key).

Some types of join operations have, by nature of the algorithm, higher memory complexity than others. However, there is also a large difference between join operations of the same type. Figure 5 shows what percentage of each type of join operation are responsible for $99 \%$ of the offchip memory accesses. Figure 5 gives three insights: (i) The 


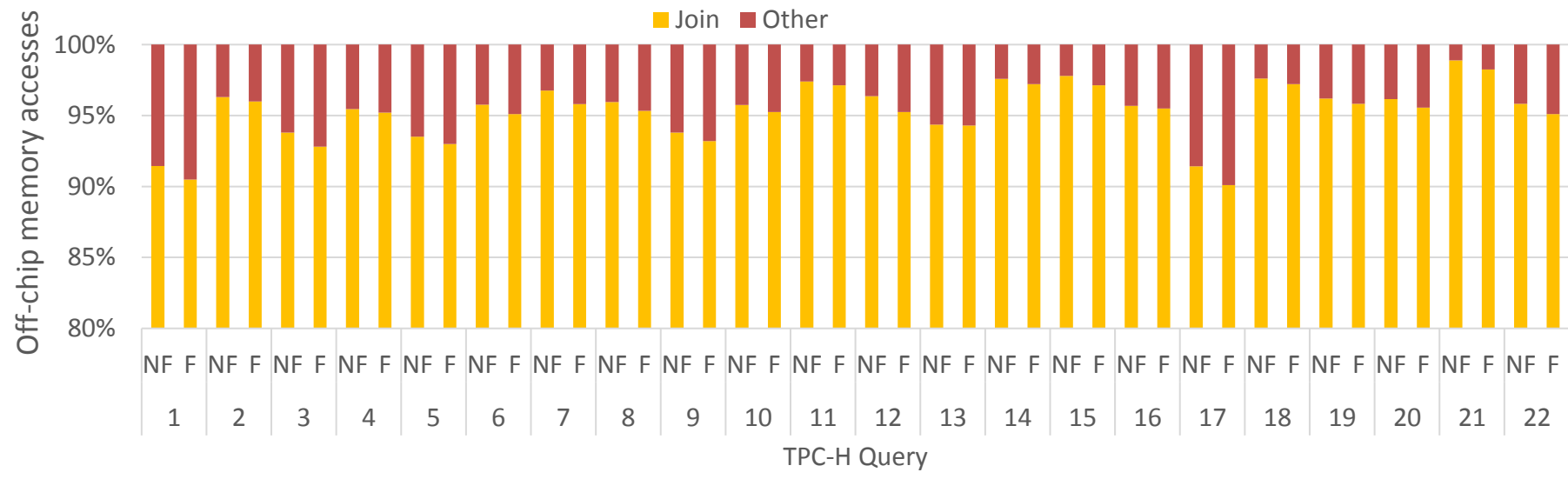

Fig. 3: Percentage of main memory accesses from join operators and other code in MonetDB Columnar Store. 'NF' corresponds to No Fragmentation and ' $F$ ' corresponds to Fragmentation.

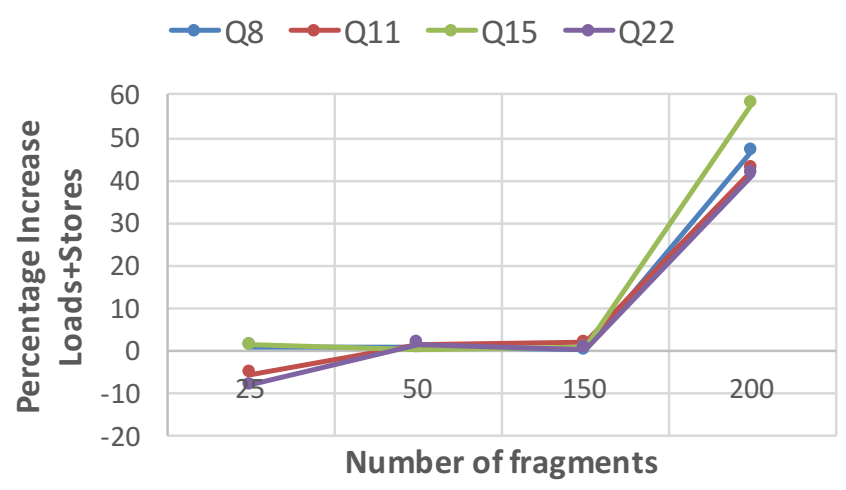

Fig. 4: The overhead of query fragmentation on 4 TPC-H queries.

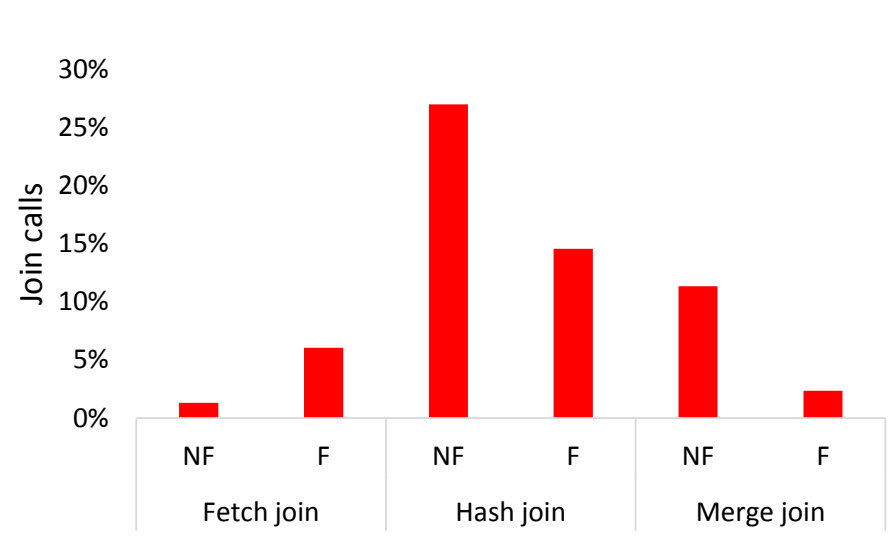

Fig. 5: The percentage of join calls serving $99 \%$ of offchip memory accesses, broken down by the type of join algorithm. 'NF' corresponds to No Fragmentation and ' $\mathrm{F}^{\prime}$ corresponds to Fragmentation.

majority of the join calls find their data in on-chip caches, which means that their operands and output data set can have NVM as a backing memory; (ii) Hash join is the most critical join among them because over $25 \%$ of the hash join calls are memory-intensive; (iii) $10 \%$ of merge joins are memory-intensive, however, this number is strongly reduced when fragmenting the query execution plan. Note that merge join operates on sorted data sets and is thus linear-time. Smaller data sets are naturally retained in cache.

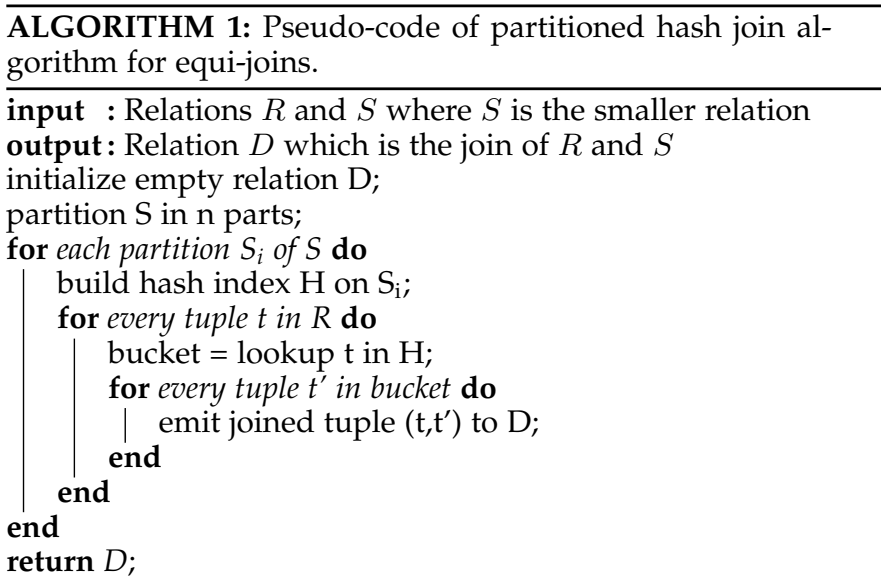

We have thus identified that the main objects in MonetDB that should be placed on DRAM are intermediate results that are used by hash join operators. Let us study the hash join operator more closely to understand its memory access patterns. Algorithm 1 depicts the structure of a partitioned hash join algorithm in pseudo-code [33]. The algorithm first partitions the smaller relation. The partitioning ensures that the algorithm has a small working set that fits in memory [26]. This is achieved by making partitions sufficiently small. For each partition, the algorithm builds an index on the join key of the smaller relation in order to efficiently retrieve tuples. Subsequently, it traverses the larger generation sequentially, looking up each tuple in the hash index. Matching tuples are then joined and emitted.

The access patterns are such that the largest relation is accessed sequentially multiple times [33], i.e., elements on subsequent memory addresses are fetched into the CPU one after another. This access pattern is the most efficient way to access memory and can exploit the highest fraction of the available memory bandwidth. The smaller relation $S$ is accessed with a random access pattern. However, by selecting the partition sizes carefully, it is possible to achieve excellent locality, hence few main memory accesses, while 
processing a partition [26], [63].

We conclude that the majority of main memory accesses in the DBMS occur while executing hash join operators and that these memory accesses are associated to the larger relation that is joined. As such, memory placement is optimized when the larger relation is placed in DRAM and the smaller relation is placed in NVM. However, when both relations are sufficiently small they will simultaneously fit in the lastlevel cache. In this case, cache misses are rare and both relations should be placed in NVM.

\section{Data Placement Heuristics}

We develop the FEED algorithm (Fast and Energy-Efficient Data placement) for placing intermediate results used by join operators in DRAM. These intermediate results can be the output of the join operation, in which case we place them on the appropriate type of memory when the join operator is initiated. Placing data on the appropriate memory type at allocation time avoids the need for data migration, which can be costly in terms of execution time and energy consumption. Intermediate results can also incur a large number of main memory accesses when they are used as inputs to the join operator. In this case, we decide on placement of the result set when it is allocated, i.e., at the start of the operator that is producing the result set.

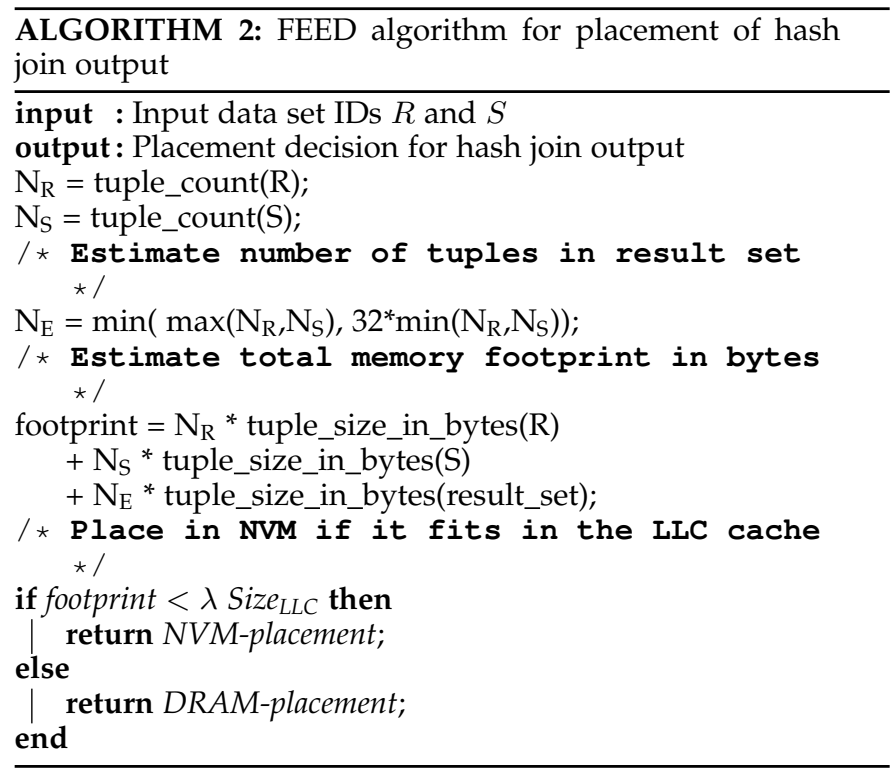

\subsection{Join Output}

Algorithm 2 shows the procedure to decide the most appropriate backing memory type for the output of the join operator. We calculate the total memory footprint of the join operator by adding the sizes of the inputs to the estimated output size. The size of an individual result set is calculated as the product of the number of tuples and the width of a tuple (how many bytes are required to store the tuple). The size estimation contains a parameter (32) that was heuristically determined to maximize the accuracy of predicting the tuple size [?]. If the memory footprint exceeds the last-level cache (LLC) size then we place the output on DRAM, else we place it on NVM. The rationale behind this heuristic is that large outputs will be re-read from DRAM, while small outputs will be re-read from the last-level cache.

FEED accounts for inaccuracy in the estimates. We decide that the footprint exceeds the LLC capacity when it is larger than a fraction $\lambda<1$ of the LLC size. The fraction $\lambda$ guards against conflict misses (as opposed to capacity misses which are determined by the size of the cache [51]). Moreover, there are other small auxiliary data structures that also occupy LLC space. We experimented with values for $\lambda$ in the range $80-100 \%$ and choose $\lambda=90 \%$. Choosing a larger value of $\lambda$ may inadvertently increase the cache miss rate and thus result in excess accesses to NVM. Choosing too small a value for $\lambda$ will under-utilize NVM and increase power consumption by reverting accesses to DRAM.

ALGORITHM 3: Placement heuristic for hash join inputs

input : Input data set IDs $R$ and $S$

output: Placement decision for $R$ given that $S$ is other input

if $S$ not allocated yet then

/* Case 1: Allocate ' $R$ ' when ' $S$ ' not yet created

$\mathrm{N}_{\mathrm{R}}$ = estimate_tuple_count $(\mathrm{R})$;

footprint $=3{ }^{*} \mathrm{~N}_{\mathrm{R}}$ * tuple_size_in_bytes $(\mathrm{R})$;

if footprint $<\lambda$ Size $_{L L C}$ then

return NVM-placement;

else

return DRAM-placement; end

else

/ Case 2: Allocate ' $R$ ' when ' $S$ ' already created

$\mathrm{N}_{\mathrm{R}}$ = estimate_tuple_count $(\mathrm{R})$;

$\mathrm{N}_{\mathrm{S}}=$ tuple_count(S);

/ * Estimate number of tuples in result set

$\mathrm{N}_{\mathrm{E}}=\min \left(\max \left(\mathrm{N}_{\mathrm{R}}, \mathrm{N}_{\mathrm{S}}\right), 32^{*} \min \left(\mathrm{N}_{\mathrm{R}}, \mathrm{N}_{\mathrm{S}}\right)\right)$;

/* Estimate total memory footprint in bytes

footprint $=\mathrm{N}_{\mathrm{R}}{ }^{*}$ tuple_size_in_bytes $(\mathrm{R})$;

$+\mathrm{N}_{\mathrm{S}}$ * tuple_size_in_bytes(S) ;

$+\mathrm{N}_{\mathrm{E}} *$ tuple_size_in_bytes(result_set);

/ * Place the smaller relation on NVM if the footprint exceeds the ILC cache capacity

if footprint $<\vec{\lambda}$ Size $_{L L C}$ then

else return $N V M$-placement;

I Place the smaller input on NVM */

if $N_{R}<N_{S}$ then

return NVM-placement;

else

| return DRAM-placement;

end

end

end

We note that in our experiments the output of the join operator may also be reused internally within the operator under specific circumstances. This effect is specific to how the internal data structures are organized in the database we experimented with. In particular, data sets are invariably represented using (a number of) arrays, each stored sequentially in memory. When these arrays are allocated, 
the database uses cost models to estimate their size. This is dependent on the query and the database content. As such, the estimate is conservative and there is a procedure to grow the arrays. The arrays grow through allocating a larger array and copying the data over. Arrays may be grown repeatedly during the execution of an operator, causing the data to be reused during execution.

Each time the output set is grown, we re-apply the heuristic of Algorithm 2 using the updated size of the output set instead of the initial estimate. As such, the output of a join operator may initially be allocated on NVM because the total footprint is small, but the output set may later grow large enough to warrant allocation on DRAM. Such migrations are rare and occur at most three per query. Note that migrating the output from NVM to DRAM incurs minimal overhead over the baseline database implementation as the operation coincides with an existing memory copy.

Implementation consideration: Copying data from NVM to DRAM may take longer compared to copying data from DRAM to DRAM. The details depend strongly on system design. On the one hand, NVM access latencies are higher than DRAM latency, which suggests that DRAM-toDRAM copy will be faster than NVM-to-DRAM copy. It is, however, likely that NVM and DRAM chips interface with a distinct memory controller due to electrical and timing differences between the memory types. As such, an NVMto-DRAM copy can avail of higher bus bandwidth and does not incur changes in bus operation (switching between read and write), which cost a few bus cycles per switch. The actual costs will become clear when hybrid memory systems become available. We make conservative assumptions in our evaluation in favor of DRAM systems.

\subsection{Join Inputs}

Most join operators access their inputs using a single sequential scan as shown in Section 4. An exception is the hash join algorithm, which uses its inputs in distinct ways. The larger input is repeatedly scanned over. For every tuple in the larger input, the appropriate key is looked up in the smaller input relation. In order to make this lookup fast, a hash index is computed on the smaller input prior to the start of the algorithm. It is furthermore possible to partition the smaller input and process one partition at a time. This minimizes cache misses and TLB misses on the hash index, but requires repeated sequential scans of the larger input [33].

Knowing this behavior of the hash join algorithm, we define a heuristic that places the larger input in DRAM and the smaller in NVM. The hash index itself has excellent locality by design of the algorithm and typically resides in the CPU cache [6]. We aim to to place data on the appropriate type of memory start at creation time. This way, we can avoid migration of data, which can negatively impact execution time and energy-efficiency. The placement heuristic (Algorithm 3) considers two cases: (i) if the total footprint of the join operator fits in the LLC, then we allocate the inputs on NVM; (ii) otherwise, we allocate the smaller input on NVM and the larger input on DRAM. In order to make these decisions, we need to estimate the memory footprint size of the join operator. The difficulty of this estimation depends on whether one or none of the join operator's inputs have already been created. We consider these cases separately.

\subsubsection{Placement of Last-Created Input}

This is Case 2 in Algorithm 3. We assume input $R$ is created and $S$ already exists. We estimate the number of tuples that $R$ will contain, depending on the properties of the operator that is generating $R$ and its inputs. Note that this operator is about to execute. As such, it has exact knowledge of its inputs. As such, we do not need to estimate result sizes recursively, which would have poor accuracy. On the basis of (estimated) input sizes for $R$ and $S$, we can then estimate the footprint of the join output using the same formula as Algorithm 2. We then proceed to place $R$ on NVM if the total footprint of the join operator fits in the cache. Otherwise, we place $R$ on NVM if it is the smaller relation.

\subsubsection{Placement of First-Created Input}

This is Case 1 in Algorithm 3. In this case we can estimate again the size of the input $R$, but for $S$ we have no information as it has not been created yet. Lacking any information, we estimate the footprint of the join result on the basis that both $S$ and the result will have the same size as $R$ (hence the factor 3). Depending on whether this footprint fits in the LLC, $R$ is placed accordingly.

Note that this heuristic works very well when the inputs have highly different sizes. Assume $R$ is allocated first. If $R$ is small, it will be allocated on NVM. If the real size of $R$ is indeed much smaller than $S$, then FEED will place $S$ on DRAM if $S$ is sufficiently large. On the other hand, if $R$ is sufficiently large it will be allocated on DRAM. If the real size of $R$ is much larger than that of $S$, then FEED follows up with allocating $S$ on NVM.

As FEED only places data and does not migrate, it requires foresight to place the inputs of the hash join operator in the correct type of memory when the inputs are created. To this end, FEED annotates the query execution plan to show every output value that is consumed by a hash join operator. The operator passes this annotation on to the FEED memory allocation algorithm when allocating memory to store its output. Annotations can be inserted in the query execution plan either during construction, or by performing a linear-time post-processing step. Every operator is represented by a node in the query execution plan. Annotations are applied by scanning the plan backwards and labeling the nodes that feed into join operators.

\subsection{Managing DRAM Contention}

DRAM contention is mitigated through query plan fragmentation. This causes intermediate data sets to be sufficiently small such that all DRAM allocations can be simultaneously satisfied. For some queries, however, this may not be sufficient. An alternative is to actively manage DRAM contention, by moving intermediate data sets between DRAM and NVM when DRAM is full. We found, however, that there is limited scope for such data movement.

Assume that at any moment in time a memory allocation is characterized by $A=(S, N)$ where $S$ is the size in bytes and $N$ is the average number of reads per byte. We 


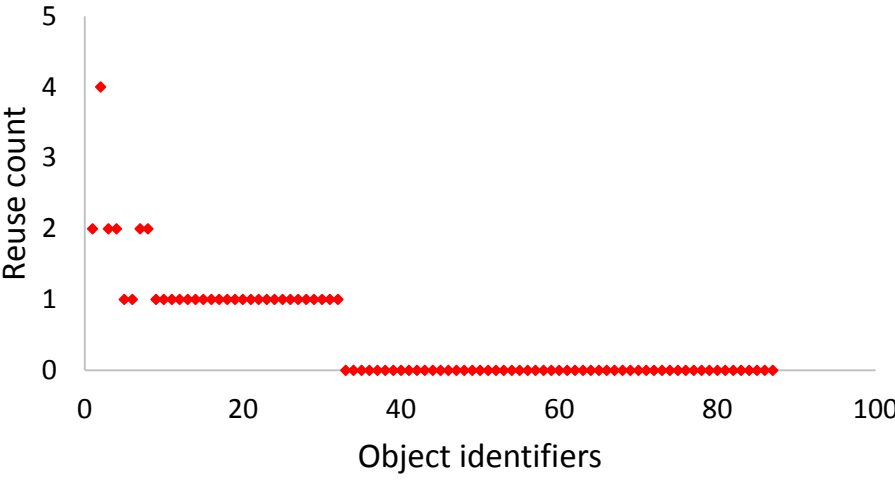

Fig. 6: Number of main memory reads per byte in each heapallocated object of TPC-H query Q11.

assume that every allocated object is write-once, which is the case for the objects we desire to place on DRAM. Assume further that allocation $A_{1}$ was made in DRAM and is a possible candidate for eviction to NVM at the time when allocation $A_{2}$ is made. We can then estimate the average memory access time (AMAT) when retaining $A_{1}$ in DRAM and allocating $A_{2}$ in NVM as

$$
N_{1} S_{1} T_{r, d r a m}+N_{2} S_{2} T_{r, n v m}+S_{2} T_{w, n v m}
$$

where $T_{r, \text { tech }}$ and $T_{w, \text { tech }}$ are the average delay of reading, respectively writing, a 64-byte block of data in memory of technology tech. The AMAT when moving $A_{1}$ to NVM can be estimated as:

$N_{1} S_{1} T_{r, n v m}+N_{2} S_{2} T_{r, d r a m}+S_{2} T_{w, d r a m}+S_{1}\left(T_{r, d r a m}+T_{w, n v m}\right)$

Moving $A_{1}$ reduces AMAT when Eq. $2<$ Eq. 1 . Assuming $S_{1}=S_{2}$ and using the technology parameters of RRAM, the best-performing NVM variant (Table 1), migration is beneficial when:

$$
64 N_{1}+120<64 N_{2}
$$

which implies that $N_{2}$ must exceed $N_{1}$ about three times or more. We measured $N$, the expected number of times a byte in an object is read from main memory during the lifetime of the object. As one can see, reuses from main memory, are infrequent. When the objects with zero reuses are read, the reads hit in the on-chip CPU caches.

As there are few case where migration is beneficial (1 object in the case of the Q11 query, see Figure 6), we decided to develop strong placement heuristics and abstain from migration. While fragmentation of queries limits intermediate data set sizes, it cannot be ruled out that an allocation cannot be served from DRAM. Such allocations, which were unobserved in our experiments, have limited impact as it is rare to re-read objects from memory frequently.

While FEED implements placement in the hybrid memory hierarchy, this does not prohibit the programmer to additionally perform migration on selected objects. As migration requires a copy of data between DRAM and NVM, it can be effected by performing a fresh memory allocation on the target memory and then copying the data over.
TABLE 2: Simulation parameters

\begin{tabular}{|l|l|}
\hline Component & configuration \\
\hline \hline LLC cache & $20 \mathrm{MB}, 4$-way set-associate, 64 byte blocks, LRU \\
\hline DRAM & $256 \mathrm{MB}, t_{R C D}=5, t_{R P}=5$, \\
& $t_{R R D p r e}=3, t_{R R D a c t}=3$ \\
\hline NVM & $8 \mathrm{~GB}$ RRAM, $t_{R C D}=10, t_{R P}=11$, \\
& $t_{R R D p r e}=6 n s, t_{R R D a c t}=6.5 \mathrm{~ns}$ \\
\hline
\end{tabular}

\section{EXPERIMENTAL EVALUATION}

\subsection{Hybrid Memory Simulator}

We use the state-of-the-art GEM5 [4] cycle accurate simulator for the validation of data placement. Table 2 summarizes the memory system properties. We configure GEM5 with two memory controllers, one interfacing with $256 \mathrm{MB}$ DRAM and one interfacing with 8GB RRAM. The timing parameters for DRAM and RRAM are derived from the Micron DDR3 datasheet [35] and prior literature [13], [28], [43]. Specifically, we derived the timing parameters for RRAM following Lee et al [28]. tRCD is the number of clock cycles required between active Row Access Strobe (RAS) and Column Access Strobe (CAS) and represents read delay. For RRAM, we configured this parameter as $2 \times$ DRAM. tRP is the number of cycles needed to terminate access to an open row and open access to the next row. tRP represents write delay. For RRAM, we configured tRP as $2.2 \times$ DRAM cycles. Moreover, we modified the GEM5 memory controller to avoid write-back of clean cache blocks to memory. This not only improves the endurance of RRAM, but also saves write energy. tRRDpre represents the delay between writes and tRRDact represents delay between reads due to power budget constraints. Following the model in [28] we derived ) tRRDpre $6 \mathrm{~ns}$ and tRRDact $6.5 \mathrm{~ns}$ for RRAM. Both DRAM and RRAM memory controllers use open page management policy with maximum 4 accesses per row before closing.

We extend GEM5 to expose the DRAM and RRAM virtual address ranges to the key memory allocation routine mmap (our malloc library builds on mmap) and to enforce the placement of global variables. We implement a new instruction in X86 instruction set which makes a special system call from an application to GEM5 in order to register starting address and size of each memory allocation. The memory controllers use this information to decide whether to send read or write operations to these ranges to DRAM or RRAM. During the simulation phase, we fast forward the first $1 \mathrm{G}$ instructions in atomic mode and simulate next 6G instructions in detailed mode with 30\% sampling rate. Performance is reported on the basis of the cycles-perinstruction metric.

In order to save simulation time and by-pass the operating system, we perform all the validation in GEM5 System Call Emulation (SE) mode. In order to run MonetDB on top of GEM5 SE mode, we extend GEM5 with missing Linux system calls such as mmap, munmap, readdir and getdents.

\subsection{Workload}

We analyze two standardized benchmarks for Decision Support Systems and Big Data [56]. TPC-H [56] models a complex decision support system. It consists of 22 analytical queries. TPC-DS [39], [57] is a more modern analytical benchmark. It contains a large number of tables and 


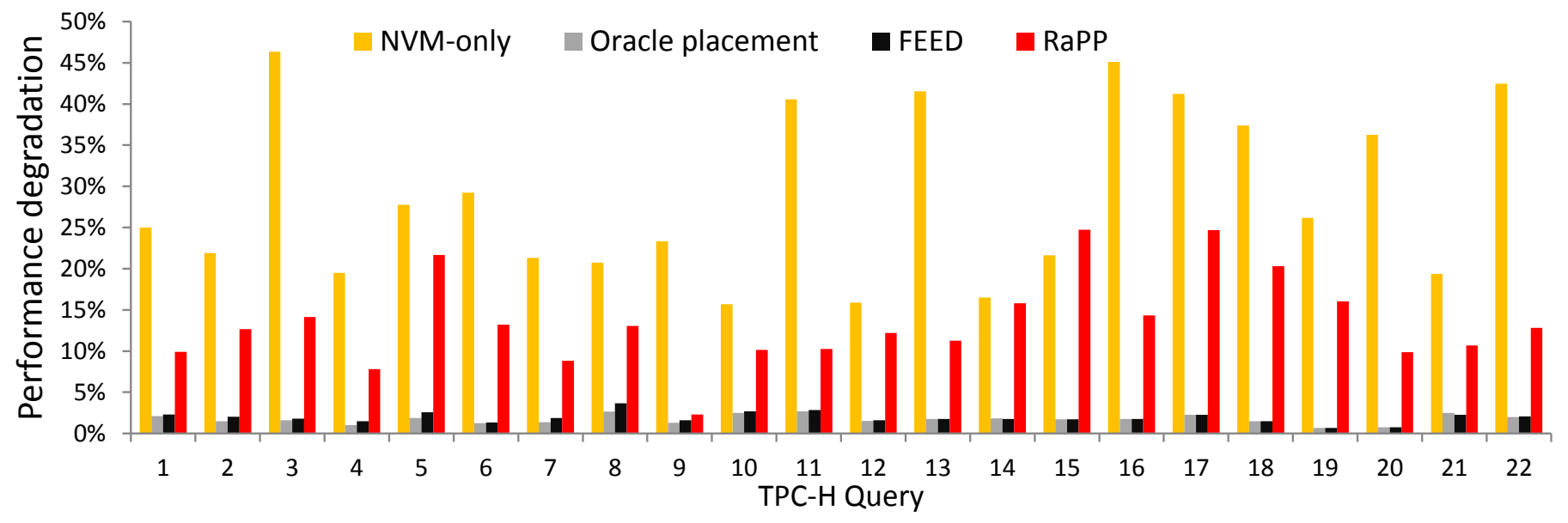

Fig. 7: TPC-H Performance degradation of an NVM-only system (NVM), oracle placement, the FEED heuristic and RaPP compared to a DRAM-only system. Lower bars are better. Cases 1-22 correspond to the 22 TPC-H queries.

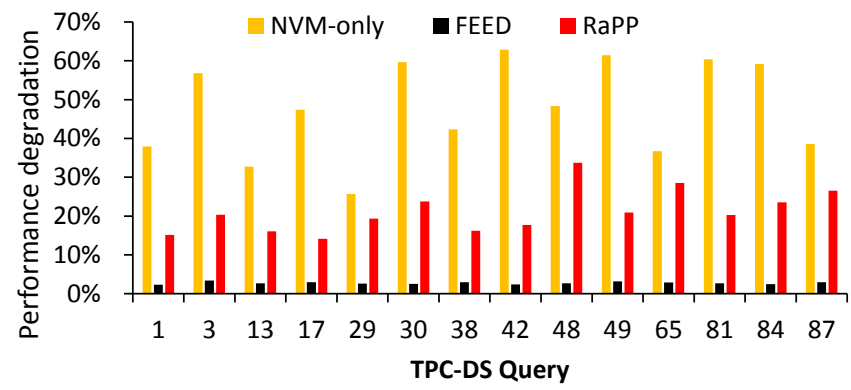

Fig. 8: TPC-DS Performance degradation of an NVM-only system (NVM), FEED and RaPP compared to a DRAM-only system. Lower bars are better. Numbered cases correspond to TPC-DS query numbers.

columns that fully test the indexing and query optimization techniques of databases for large data sets. It consists of 99 analytical queries. We use these benchmarks at scale factor 5.0. We demonstrate our memory management techniques in the MonetDB database [7]. We compile MonetDB from source with the CLANG compiler and '-O2' optimization.

\subsection{Application Data Placement}

We implement a custom memory allocator for DRAM and NVM by extending the dlmalloc memory allocator ${ }^{1}$, version 2.8.6. We build two custom memory allocators, one each for DRAM and NVM. Both allocators use LINUX memory management ${ }^{2}$ to allocate large distinct virtual memory address (VMA) regions. In a 64 bit OS, 48 bits are used for virtual address. The number of available virtual addresses in 64 bit OS are $2^{48}$ which is more than what is needed in the existing systems. Large part of that address space remain un-used. We use this un-used virtual address space to allocate two distinct regions for DRAM and NVM and let the custom memory allocators manage those explicitly to serve memory allocation requests from the application. The custom allocators claim distinct virtual memory address regions for each memory type using the MAP Fixed feature

1. ftp://gee.cs.oswego.edu/pub/misc/malloc.c

2. http://www.makelinux.net/ldd3/chp-15-sect-1 of the Linux mmap system call which allows us to control the starting address of each region.

\subsection{Impact on Performance}

For TPC-H, Figure 7 shows the performance of our heuristic placement policy called FEED. It compares this against 3 other configurations: an NVM-only system, a hybrid system with oracle based quantitative placement methodology and a hybrid system with the RaPP hardware scheme. Performance shown is performance degradation over a DRAMonly system, so lower bars are better.

The oracle scheme is explained in Appendix ??. It represents the best possible decision of allocations that can be made, using perfect foresight of how many main memory accesses will occur. The performance is measured in the unit of cycles per instructions (CPI). Clearly, the NVM-only system has the worst performance because of the latency difference between DRAM and NVM. Note that the main difference between the NVM-only and other techniques is that the NVM-only solution places the main memory accesses that are most sensitive to memory latency on the slowest memory type. This has a major impact on performance. The performance decrease depends on the number of off-chip accesses made by each individual query. TPC-H Q3 and Q16 have the worst impact on performance due to slow memory. However, the oracle placement (static placement) is based on a static placement model which keeps the performance decrease within a programmer defined value. We set it to $5 \%$ in this paper. The results confirm that the oracle placement is effective in hiding the higher latencies of NVM, however it is a static approach which does not suite the dynamic nature of database workloads. Contrary to this, FEED perform equally well as oracle placement. In fact TPC$\mathrm{H}$ Q21 has lesser CPI decrease for FEED in comparison to oracle placement. Finally, RaPP doesn't perform well in hiding the latencies of NVM. For TPC-H Q9, RaPP is closer to FEED but for the rest of the queries the average increase in CPI is greater than 10 which is very high. We analyze MonetDB at the granularity of OS virtual page to understand why RaPP does not perform well. RaPP is based on multilevel queues where the pages are assigned some 


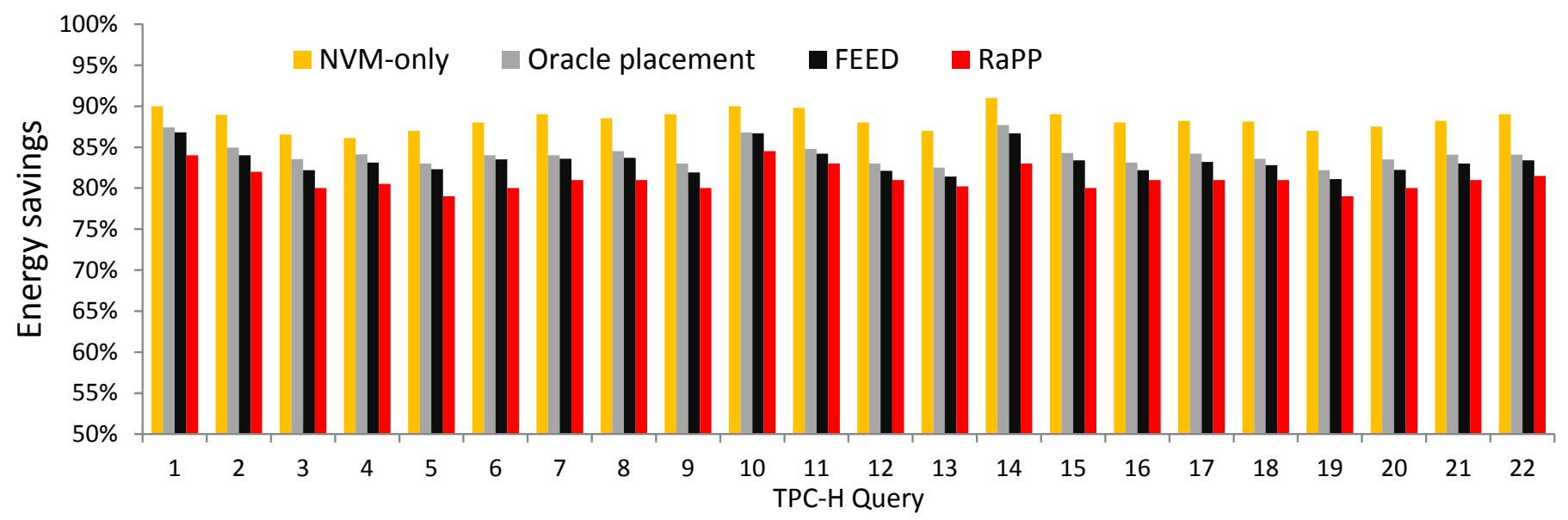

Fig. 9: TPC-H Energy reduction of an NVM-only system (NVM), oracle placement, FEED and RaPP compared to a DRAMonly system. Higher bars are better. Cases 1-22 correspond to the 22 TPC-H queries.

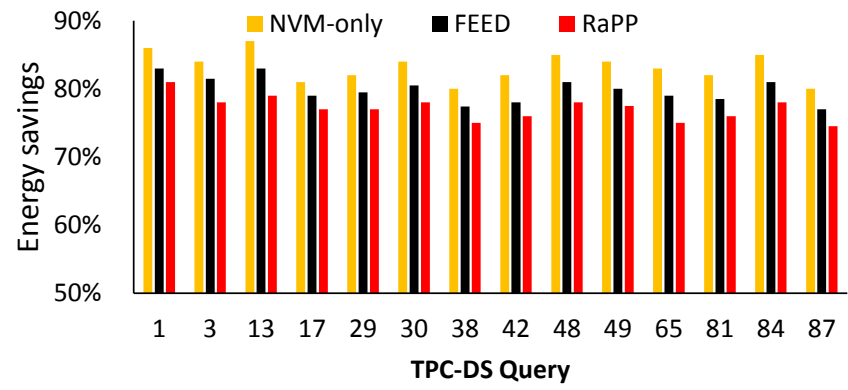

Fig. 10: TPC-DS Energy reduction of an NVM-only system (NVM), FEED and RaPP compared to a DRAM-only system. Higher bars are better. Numbered cases correspond to TPCDS query numbers.

rank to place them on DRAM queue or NVM queue. Our analysis reveals that MonetDB has highly cache conscious algorithms and the off-chip accesses to the virtual pages are scattered. Consequently, there are not enough memory accesses to lot of pages in order to rank them precisely in RaPP. Moreover, RaPP incurs migration cost and uses second-guess approach. Whereas FEED is based on intuition and takes proactive decision on data placement by using the application knowledge.

For TPC-DS, Figure 8 summarizes the performance of NVM-only system, hybrid memory with our 'FEED' approach and hybrid system with RaPP in comparison to a DRAM-only system. The performance decrease is relative to the number of off-chip accesses made by each individual query. Similar to TPC-H, NVM-only system has the worst performance for all the TPC-DS queries in comparison to DRAM-only system. The maximum performance decrease is $62 \%$ for TPCH-DS Q42. RaPP does not perform well either for any TPC-DS query. Q48 shows the maximum performance degradation up to $34 \%$. FEED performs the best of all with the performance degradation less than $3.3 \%$ for all the TPC-DS queries (see Figure 8).

\subsubsection{Analysis of Energy Savings}

Our analysis shows that, for a column-oriented database, static energy dominates dynamic energy. More than $95 \%$ of the total energy is the static energy for TPC-H workload for scale factor 5 running on MonetDB with underlying memory system of size $8 \mathrm{~GB}$. Consequently, the different in static energy dictates the energy savings in hybrid memory system regardless of the dynamic energy. The increase in dynamic energy due to NVM does not change the overall energy savings by much as shown in our results. Figure 9 shows the energy savings of NVM-only system, oracle placement, FEED and RaPP in comparison to DRAM-only system. NVM-only system has the highest energy savings because static energy of NVM is significantly lower than DRAM. However, NVM-only system performs worse in terms of performance. So the NVM-only system cannot be practical. On the other hand, oracle placement and heuristics have slightly less energy savings than the NVM-only system but these solutions ensure that the performance degradation is less than $5 \%$ as shown in figure 7. RaPP performs worse in energy savings because more pages are placed on NVM by the ranking algorithm and energy consumed during migration of pages between memories.

Similarly for TPC-DS workload, Figure 10 shows the energy savings of NVM-only system, FEED and RaPP in comparison to DRAM-only system. NVM-only system gives the highest energy savings but at the expense of increase CPI (see Figure 8). However, FEED gives energy savings up to $81.5 \%$ with maximum performance degradation up to $3.3 \%$. RaPP does not perform well for energy savings in contrast to FEED approach (Figure 10).

\subsubsection{DRAM vs NVM Reads and Writes}

Figure 11 demonstrates the effectiveness of FEED to direct memory accesses to DRAM. It shows what percentage of the read, respectively write, accesses to main memory are serviced from DRAM. The remainder are serviced from NVM. At least $83 \%$ and up to $98 \%$ of the main memory accesses are mapped to DRAM across all queries. This high accuracy explains why our FEED approach achieve performance and energy close to the oracle.

Figure 11 moreover illustrates how the varying working set sizes for the queries affects the fraction of memory accesses that are served from DRAM. When the working 


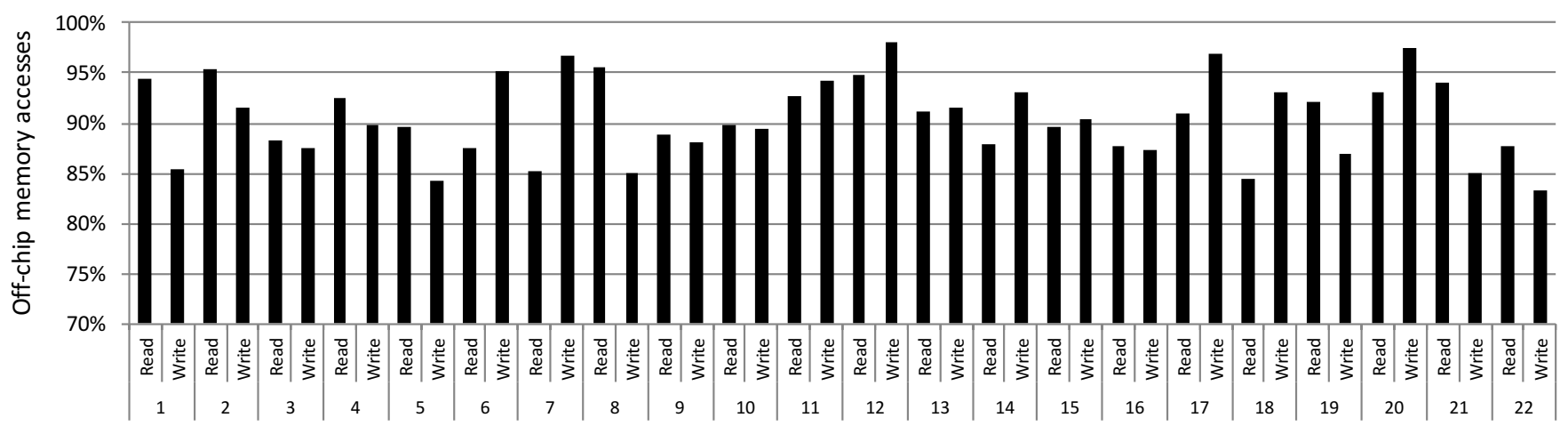

Fig. 11: Percentage of read and write, main memory accesses that are serviced from DRAM in the modified MonetDB.

set size is larger, a lesser fraction of DRAM objects fits in DRAM and more memory accesses are served from NVM.

It is worth pointing out that servicing all memory accesses from DRAM may not be an optimal configuration, as the bandwidth of NVM would be unutilized. This problem was analyzed for a tiered memory system with near (3D diestacked) and far DRAM memory [10]. A system is proposed whereby part of the near memory is disabled in order to force more accesses to the far memory. Bandwidth concerns were not included in the design of FEED, but could prove an interesting topic for future research.

\subsection{Ease of Implementation}

There are around 3250 dynamic allocation call sites in MonetDB that use malloc or mmap. Our default policy is to use NVM. There are only 7 call sites where a decision must be made to allocate on DRAM or NVM for OLAP workloads. As such, we were able to adapt a state-of-the-art database to hybrid memory with minimal code changes.

\section{Conclusion}

Non-volatile memory is crucial in order to sustain growing data sets for in-memory data intensive computing. As NVM has lower performance and higher energy consumption on access compared to DRAM, it is necessary to build a hybrid main memory system. This paper explores how databases can control the placement of data in either DRAM or NVM in order to optimize performance and minimize energy consumption for analytic workloads.

We have performed an in-depth analysis of a state-ofthe-art column-oriented database and found that materialized intermediate data sets are the major contributor of total off-chip accesses. We have demonstrated that joins in general and hash-join in particular are the most critical database operators that cause most of the accesses to main memory for standardized OLAP benchmarks.

We propose online heuristics for dynamic allocation of the inputs and the output of critical join operators. Using cycle accurate simulation, we demonstrate that heuristic placement is as good as static oracle placement and much better than state-of-the-art application-agnostic data migration techniques. Only a handful of memory allocation sites in the DBMS need to be modified to allocate data sets on
DRAM. The remainder of the allocations use the default allocator, mapping data onto NVM.

This work is a first step in exploring application-specific data placement in hybrid memories. We have made minimal adjustments to the MonetDB database to demonstrate the feasibility of this approach, however, further optimizations will enhance the effectiveness of the proposed techniques, e.g., query plan optimization may reduce the lifetime of fragments, fragmentation should control the size of the intermediate and depend on the memory type holding the intermediate. Moreover, it is an open question how much DRAM cache storage is required for effective operation. This will likely vary with the total database size and with the amount of NVM.

The techniques developed in this work apply to many types of hybrid memory. DRAM+NVM and die-stacked memory [5] are just two design points that will be realized in the near future. Future work is to apply our approach to other workloads where other operations, such as sorting and aggregation, may become more important bottlenecks.

\section{ACKNOWLEDGMENTS}

This work is supported by the European Community's Seventh Framework Programme (FP7/2007-2013) under the NovoSoft project (Marie Curie Actions, grant agreement 327744) and the NanoStreams project (grant agreement 610509)

\section{REFERENCES}

[1] D. J. Abadi, D. S. Myers, D. J. DeWitt, and S. R. Madden. Materialization strategies in a column-oriented dbms. In 2007 IEEE $23 \mathrm{rd}$ International Conference on Data Engineering, pages 466-475, April 2007.

[2] J. Arulraj, A. Pavlo, and S. R. Dulloor. Let's talk about storage \& recovery methods for non-volatile memory database systems. In Proceedings of the 2015 ACM SIGMOD International Conference on Management of Data, SIGMOD '15, pages 707-722, New York, NY, USA, 2015. ACM.

[3] L. A. Barroso and U. Hölzle. The datacenter as a computer: An introduction to the design of warehouse-scale machines. Synthesis Lectures on Computer Architecture, 4(1):1-108, 2009.

[4] N. Binkert, B. Beckmann, G. Black, S. K. Reinhardt, A. Saidi, A. Basu, J. Hestness, D. R. Hower, T. Krishna, S. Sardashti, R. Sen, K. Sewell, M. Shoaib, N. Vaish, M. D. Hill, and D. A. Wood. The gem5 simulator. SIGARCH Comput. Archit. News, 39(2):1-7, Aug. 2011. 
[5] B. Black, M. Annavaram, N. Brekelbaum, J. DeVale, L. Jiang, G. H. Loh, D. McCaule, P. Morrow, D. W. Nelson, D. Pantuso, P. Reed, J. Rupley, S. Shankar, J. Shen, and C. Webb. Die stacking (3d) microarchitecture. In Proceedings of the 39th Annual IEEE/ACM International Symposium on Microarchitecture, MICRO 39, pages 469-479, Washington, DC, USA, 2006. IEEE Computer Society.

[6] P. A. Boncz, M. L. Kersten, and S. Manegold. Breaking the memory wall in monetdb. Commun. ACM, 51(12):77-85, Dec. 2008.

[7] P. A. Boncz, S. Manegold, and M. L. Kersten. Database architecture optimized for the new bottleneck: Memory access. In Proceedings of the 25th International Conference on Very Large Data Bases, VLDB '99, pages 54-65, San Francisco, CA, USA, 1999. Morgan Kaufmann Publishers Inc.

[8] G. Burr, B. Kurdi, J. Scott, C. Lam, K. Gopalakrishnan, and R. Shenoy. Overview of candidate device technologies for storageclass memory. IBM Journal of Research and Development, 52(4.5):449464, July 2008.

[9] S. Cho, P.-C. Yew, and G. Lee. Decoupling local variable accesses in a wide-issue superscalar processor. In Proceedings of the 26th Annual International Symposium on Computer Architecture, ISCA '99, pages 100-110, Washington, DC, USA, 1999. IEEE Computer Society.

[10] C. Chou, A. Jaleel, and M. Qureshi. BATMAN: Techniques for maximizing system bandwidth of memory systems with stackeddram. In Proceedings of the International Symposium on Memory Systems, MEMSYS '17, pages 268-280, New York, NY, USA, 2017. ACM.

[11] J. Coburn, A. M. Caulfield, A. Akel, L. M. Grupp, R. K. Gupta, R. Jhala, and S. Swanson. NV-Heaps: making persistent objects fast and safe with next-generation, non-volatile memories. In Proceedings of the sixteenth international conference on Architectural support for programming languages and operating systems, ASPLOS XVI, pages 105-118, New York, NY, USA, 2011. ACM.

[12] G. Dhiman, R. Ayoub, and T. Rosing. PDRAM: A hybrid PRAM and DRAM main memory system. In Proceedings of the 46th Annual Design Automation Conference, DAC '09, pages 664-469, New York, NY, USA, 2009. ACM.

[13] E. Doller. Forging a future in memory - new technologies, new markets, new applications, 2010.

[14] X. Dong, C. Xu, Y. Xie, and N. Jouppi. NVSim: A circuit-level performance, energy, and area model for emerging nonvolatile memory. Computer-Aided Design of Integrated Circuits and Systems, IEEE Transactions on, 31(7):994-1007, July 2012.

[15] V. V. Fedorov, S. Qiu, A. L. N. Reddy, and P. V. Gratz. Ari: Adaptive llc-memory traffic management. ACM Trans. Archit. Code Optim., 10(4):46:1-46:19, Dec. 2013.

[16] J. Guerra, L. Mármol, D. Campello, C. Crespo, R. Rangaswami, and J. Wei. Software persistent memory. In Proceedings of the 2012 USENIX conference on Annual Technical Conference, USENIX ATC'12, pages 29-29, Berkeley, CA, USA, 2012. USENIX Association.

[17] A. Hassan, H. Vandierendonck, and D. S. Nikolopoulos. Energyefficient in-memory data stores on hybrid memory hierarchies. In Proceedings of the 11th International Workshop on Data Management on New Hardware, DaMoN'15, pages 1:1-1:8, New York, NY, USA, 2015. ACM

[18] A. Hassan, H. Vandierendonck, and D. S. Nikolopoulos. Softwaremanaged energy-efficient hybrid DRAM/NVM main memory. In Proceedings of the 12th ACM International Conference on Computing Frontiers, CF '15, pages 23:1-23:8, New York, NY, USA, 2015. ACM.

[19] Y. Ho, G. M. Huang, and P. Li. Nonvolatile memristor memory: Device characteristics and design implications. In Proceedings of the 2009 International Conference on Computer-Aided Design, ICCAD '09, pages 485-490, New York, NY, USA, 2009. ACM.

[20] IDC big data and business analytics forum 2013. https://www.idc.com/cee/events/54189-idc-big-data-andbusiness-analytics-forum-2013, 2013.

[21] S. Idreos, F. Groffen, N. Nes, S. Manegold, S. Mullender, M. Kersten, et al. Monetdb: Two decades of research in column-oriented database architectures. Bulletin of the IEEE Computer Society Technical Committee on Data Engineering, 35(1):40-45, 2012.

[22] ITRS. International Technology Roadmap for Semiconductors, 2011 Edition, Emerging Research Devices (ERD), 2011.

[23] M. Ivanova, M. Kersten, and F. Groffen. Just-in-time data distribution for analytical query processing. In Proceedings of the 16th East European Conference on Advances in Databases and Information Sys- tems, ADBIS'12, pages 209-222, Berlin, Heidelberg, 2012. SpringerVerlag.

[24] A. Jaleel, K. B. Theobald, S. C. Steely, Jr., and J. Emer. High performance cache replacement using re-reference interval prediction (rrip). In Proceedings of the 37th Annual International Symposium on Computer Architecture, ISCA '10, pages 60-71, New York, NY, USA, 2010. ACM.

[25] High Bandwidth Memory (HBM) DRAM, Oct. 2013. JESD235.

[26] M. Kitsuregawa, H. Tanaka, and T. Moto-Oka. Application of hash to data base machine and its architecture. New Generation Computing, 1(1):63-74, 1983.

[27] E. Kultursay, M. Kandemir, A. Sivasubramaniam, and O. Mutlu. Evaluating STT-RAM as an energy-efficient main memory alternative. In Performance Analysis of Systems and Software (ISPASS), 2013 IEEE International Symposium on, pages 256-267, 2013.

[28] B. C. Lee, E. Ipek, O. Mutlu, and D. Burger. Architecting phase change memory as a scalable dram alternative. In Proceedings of the 36th annual international symposium on Computer architecture, ISCA '09, pages 2-13, New York, NY, USA, 2009. ACM.

[29] H.-H. S. Lee and G. S. Tyson. Region-based caching: An energydelay efficient memory architecture for embedded processors. In Proceedings of the 2000 International Conference on Compilers, Architecture, and Synthesis for Embedded Systems, CASES '00, pages 120-127, New York, NY, USA, 2000. ACM.

[30] C. Lefurgy, K. Rajamani, F. Rawson, W. Felter, M. Kistler, and T. Keller. Energy management for commercial servers. Computer, 36(12):39-48, 2003.

[31] Y. Li, Y. Chen, and A. K. Jones. A software approach for combating asymmetries of non-volatile memories. In Proceedings of the 2012 ACM/IEEE International Symposium on Low Power Electronics and Design, ISLPED '12, pages 191-196, New York, NY, USA, 2012. ACM.

[32] The LLVM compiler infrastructure. http://llvm.org.

[33] S. Manegold, P. Boncz, and M. Kersten. Optimizing main-memory join on modern hardware. IEEE Transactions on Knowledge and Data Engineering, 14(4):709-730, Jul 2002.

[34] D. Meisner, B. T. Gold, and T. F. Wenisch. Powernap: eliminating server idle power. In Proceedings of the 14th international conference on Architectural support for programming languages and operating systems, ASPLOS XIV, pages 205-216, New York, NY, USA, 2009. ACM.

[35] Micron TN-41-01: Calculating memory system power. http://www.micron.com/products/support/power-calc, 2007. Last consulted: Nov 2014.

[36] A. Mirhoseini, M. Potkonjak, and F. Koushanfar. Coding-based energy minimization for phase change memory. In Proceedings of the 49th Annual Design Automation Conference, DAC '12, pages 6876, New York, NY, USA, 2012. ACM.

[37] J. C. Mogul, E. Argollo, M. Shah, and P. Faraboschi. Operating system support for nvm+dram hybrid main memory. In Proceedings of the 12th Conference on Hot Topics in Operating Systems, HotOS'09, pages 14-14, Berkeley, CA, USA, 2009. USENIX Association.

[38] Monetdb: The column-store pioneer. https://www.monetdb.org/Home.

[39] R. O. Nambiar and M. Poess. The making of TPC-DS. In Proceedings of the 32Nd International Conference on Very Large Data Bases, VLDB '06, pages 1049-1058. VLDB Endowment, 2006.

[40] I. Oukid, D. Booss, W. Lehner, P. Bumbulis, and T. Willhalm. Sofort: A hybrid scm-dram storage engine for fast data recovery. In Proceedings of the Tenth International Workshop on Data Management on New Hardware, DaMoN '14, pages 8:1-8:7, New York, NY, USA, 2014. ACM.

[41] I. Oukid, W. Lehner, T. Kissinger, T. Willhalm, and P. Bumbulis. Instant recovery for main memory databases. In CIDR 2015, Seventh Biennial Conference on Innovative Data Systems Research, Asilomar, CA, USA, January 4-7, 2015, Online Proceedings, 2015.

[42] S. Pelley, T. F. Wenisch, B. T. Gold, and B. Bridge. Storage management in the nvram era. Proc. VLDB Endow., 7(2):121-132, Oct. 2013.

[43] T. Perez and C. De Rosa. Non-volatile memory: Emerging technologies and their impacts on memory systems. Technical report, Technical report, 2010.

[44] pmem.io: Persistent memory programming. http://pmem.io/, 2015.

[45] M. K. Qureshi, A. Jaleel, Y. N. Patt, S. C. Steely, and J. Emer. Adaptive insertion policies for high performance caching. In Proceedings of the 34th Annual International Symposium on Computer 
Architecture, ISCA '07, pages 381-391, New York, NY, USA, 2007. ACM.

[46] M. K. Qureshi, V. Srinivasan, and J. A. Rivers. Scalable high performance main memory system using phase-change memory technology. In Proceedings of the 36th annual international symposium on Computer architecture, ISCA '09, pages 24-33, New York, NY, USA, 2009. ACM.

[47] L. E. Ramos, E. Gorbatov, and R. Bianchini. Page placement in hybrid memory systems. In Proceedings of the international conference on Supercomputing, ICS '11, pages 85-95, New York, NY, USA, 2011. ACM.

[48] SAP + Google + Intel: The winning formula for processing more data at speed with lower TCO. https://news.sap.com/2018/07/sap-google-intel-process-moredata-lower-tco/, 2018.

[49] S. Schuh, X. Chen, and J. Dittrich. An experimental comparison of thirteen relational equi-joins in main memory. In Proceedings of the 2016 International Conference on Management of Data, SIGMOD '16, pages 1961-1976, New York, NY, USA, 2016. ACM.

[50] D.-J. Shin, S. K. Park, S. M. Kim, and K. H. Park. Adaptive page grouping for energy efficiency in hybrid PRAM-DRAM main memory. In Proceedings of the 2012 ACM Research in Applied Computation Symposium, RACS '12, pages 395-402, New York, NY, USA, 2012. ACM.

[51] A. J. Smith. Cache memories. ACM Computing Surveys, 14(3):473530, Sept. 1982.

[52] A. J. Smith. Line (block) size choice for CPU cache memories. IEEE Transactions on Computers, 36(9):1063-1075, Sept. 1987.

[53] A. Sodani, R. Gramunt, J. Corbal, H. S. Kim, K. Vinod, S. Chinthamani, S. Hutsell, R. Agarwal, and Y. C. Liu. Knights landing: Second-generation intel xeon phi product. IEEE Micro, 36(2):34-46, Mar 2016.

[54] S. Srinivasan. Prefetching Vs The Memory System : Optimizations for Multi-core Server Platforms. PhD thesis, Electrical and Computer Engineering, University of Maryland, Oct. 2007.

[55] M. Stonebraker, D. J. Abadi, A. Batkin, X. Chen, M. Cherniack, M. Ferreira, E. Lau, A. Lin, S. Madden, E. O'Neil, P. O'Neil, A. Rasin, N. Tran, and S. Zdonik. C-store: A column-oriented dbms. In Proceedings of the 31st International Conference on Very Large Data Bases, VLDB '05, pages 553-564. VLDB Endowment, 2005.

[56] Transaction processing performance council. http://www.tpc.org.

[57] TPC benchmark ${ }^{\mathrm{TM}} \mathrm{DS}$ (TPC-DS): The benchmark standard for decision support solutions including big data. http://www.tpc.org/tpcds/default.asp.

[58] H. Vandierendonck, A. Hassan, and D. Nikolopoulos. On the energy-efficiency of byte-addressable non-volatile memory. Computer Architecture Letters, PP(99):1-1, 2014.

[59] Z. Wang, Z. Gu, and Z. Shao. Optimizated allocation of data variables to PCM/DRAM-based hybrid main memory for realtime embedded systems. Embedded Systems Letters, IEEE, 6(3):6164, Sept 2014.

[60] Z. Wang, S. Shan, T. Cao, J. Gu, Y. Xu, S. Mu, Y. Xie, and D. A. Jiménez. Wade: Writeback-aware dynamic cache management for nvm-based main memory system. ACM Trans. Archit. Code Optim., 10(4):51:1-51:21, Dec. 2013.

[61] W. Wei, D. Jiang, S. A. McKee, J. Xiong, and M. Chen. Exploiting program semantics to place data in hybrid memory. In Proceedings of the 24th International Conference on Parallel Architectures and Compilation Techniques (PACT), San Francisco, CA, Oct. 2015.

[62] H. Yoon. Row buffer locality aware caching policies for hybrid memories. In Proceedings of the 2012 IEEE 30th International Conference on Computer Design (ICCD 2012), ICCD '12, pages 337-344, Washington, DC, USA, 2012. IEEE Computer Society.

[63] H. Zeller and J. Gray. An adaptive hash join algorithm for multiuser environments. In Proceedings of the 16th International Conference on Very Large Data Bases, VLDB '90, pages 186-197, San Francisco, CA, USA, 1990. Morgan Kaufmann Publishers Inc.

[64] P. Zhou, B. Zhao, J. Yang, and Y. Zhang. A durable and energy efficient main memory using phase change memory technology. In Proceedings of the 36th annual international symposium on Computer architecture, ISCA '09, pages 14-23, New York, NY, USA, 2009. ACM.

[65] O. Zilberberg, S. Weiss, and S. Toledo. Phase-change memory: An architectural perspective. ACM Comput. Surv., 45(3):29:1-29:33, July 2013.

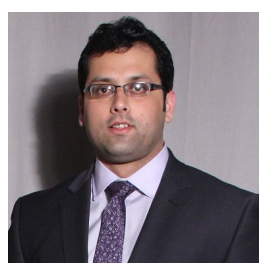

Ahmad Hassan Ahmad Hassan is a senior software Architect in SAP UK Limited. He is currently working on SAP Commerce Cloud with main emphasis on commercialisation of Context Driven Services to SAP customers. SAP Commerce Cloud provides personalisation of commerce experience by capturing and analysing every moment of user behaviour. His work is to ensure that the product is designed as Cloud Microservices based system running over Kubernetes and scale well with growing number of customers sending millions of merchandising events per day on SAP Commerce Cloud. He holds a PhD in Computer Science and has more than 10 years of experience in working for prestigious organisations like CERN, HP, Google and SAP.

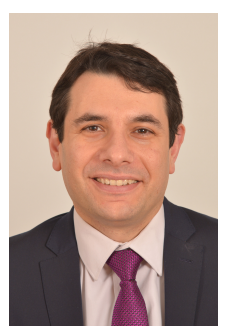

Dimitrios S. Nikolopoulos Dimitrios Nikolopoulos is Professor in the School of Electronics, Electrical Engineering and Computer Science and Director of the Institute of Electronics, Communications and Information Technology (ECIT) at Queen's University Belfast. His research interests are in system software, architecture, and performance engineering of large-scale computing systems. He serves on the Strategic Advisory Board on elnfrastructure for the UK Engineering and Physical Sciences Research Council. He holds a Royal Society Wolfson Research Merit Award and is Distinguished Member of the ACM and Senior Member of the IEEE. He completed his $\mathrm{PhD}$ in 2000 at the University of Patras.

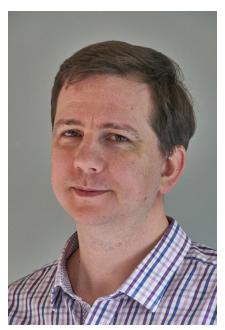

Hans Vandierendonck Hans Vandierendonck is Associate Professor (Senior Lecturer) in the School of Electronics, Electrical Engineering and Computer Science, and a Fellow of the Institute on Electronics, Communications and Information Technology at Queen's University Belfast. His research aims to build efficient and scalable computing systems for data-intensive applications. $\mathrm{He}$ is a Senior Member of the IEEE and a Senior Member of the ACM. He holds a PhD degree from Ghent University. 